\title{
Adenocarcinoma In Situ in Tubular Adenoma
}

National Cancer Institute

\section{Source}

National Cancer Institute. Adenocarcinoma In Situ in Tubular Adenoma. NCI Thesaurus.

Code C7679.

An intraepithelial adenocarcinoma arising from a tubular adenoma. 